\title{
Digital and silicone impressions vs digital calipers
}

\author{
Dora Kishkilova $^{1}$, Rosen Borisov ${ }^{1}$
}

1. Department of Diagnostic Imaging and Oral Diagnostic Faculty of Dentistry Medical University Sofia;

\begin{abstract}
Our objectives were to evaluate and compare the reliability of digital and silicone impression measurements compared to direct manual measurement performed with digital caliper. Methods: 48 simulated lesions in the upper and lowers jaws of 6 porcine heads with were used. The jaws were digitized using intraoral scanner 3SHAPE TRIOS. Digitized 3-dimensional models were converted to individual stereolithography files and used with commercial software to obtain the diameter and depth measurements of each lesion. Manual measurements were carried out with a digital caliper. Silicone impressions were made for each jaw and lesion and scanned with IOS. One-sample t-tests and linear regression analyses were performed. To further graphically examine the accuracy of the different methods, Bland-Altman plots were used. Results: We found significant differences between the diameter $M d+M x$ measured with caliper and digitalized silicone impressions. There was fixed bias between depth Mx with IOS and digital caliper. Bland-Altman analysis showed fixed bias of one approach vs the other for the depths of lesions Fi3sh for both jaws. Conclusions: Intraoral scanner is proved to be a reliable method for linear measurements of simulated lesion in the jaws. Silicone impressions are not suitable for assessing accuracy of CBCT linear measurements.
\end{abstract}

Keywords: 3Shape, silicone impressions, СВCT, accuracy

\section{Introduction}

The growing application of CBCT in dental medicine has also led to a growing interest in the accuracy of the method for taking linear and volumetric measurements. In relevant research, we found three 
types of physical measurements accepted as the "gold standard" in the evaluation of the accuracy of radiographic СBCT measurements: direct measurement with digital caliper, silicone impressions of simulated lesions and intraoral optical scanners (IOS). In our study, we tried to examine the reliability of different physical methods used in the analysis of the accuracy of CBCT measurements.

\section{Materials and methods}

e used 48 simulated lesions in the mandible $(\mathrm{Md})$ and maxilla $(\mathrm{Mx})$ of six porcine heads. We performed direct triple measuremnets (Fi) of the diameter and depth of each lesion (fig.1) with a digital caliper and compared the results with the corresponding measurements (Fsi) on silicone (Fig. 2,3,4) and digital impressions (F3sh) obtained by IOS (Fig.5).

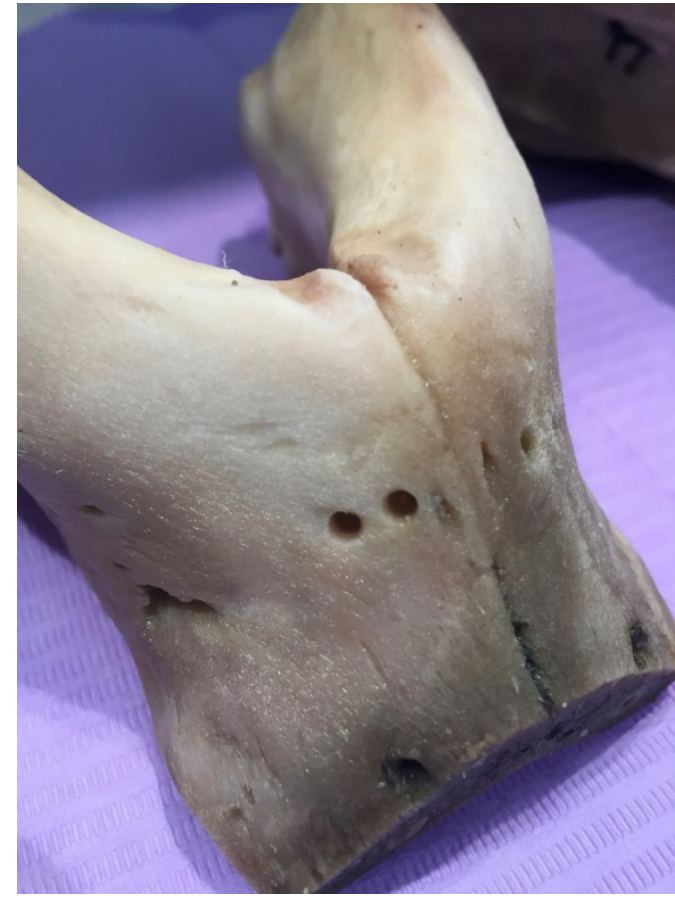

a.

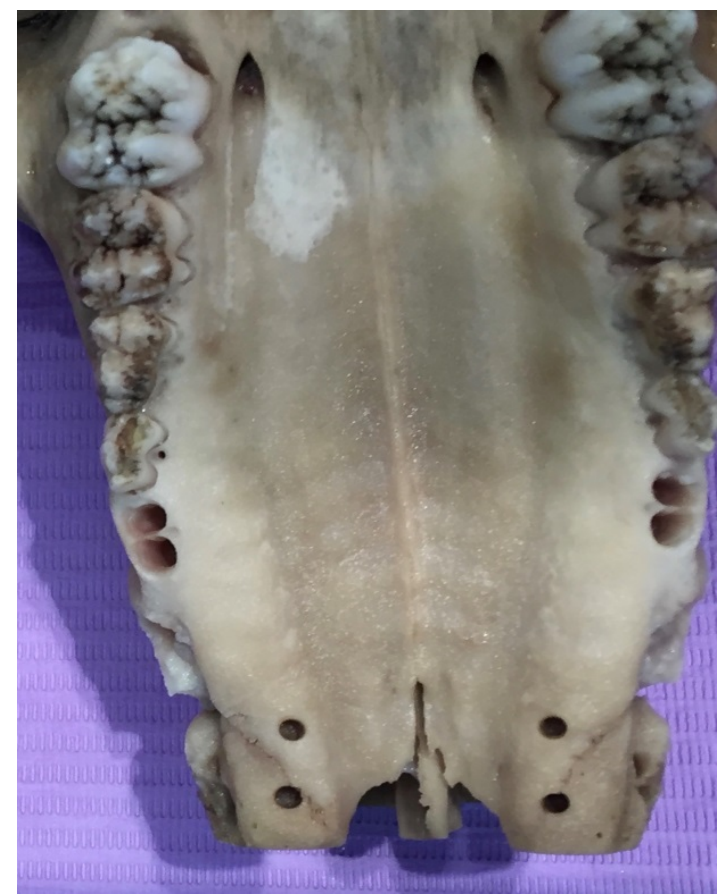

b.

Fig.1 Simulated lesions in the mandible (a) and maxilla (b) 

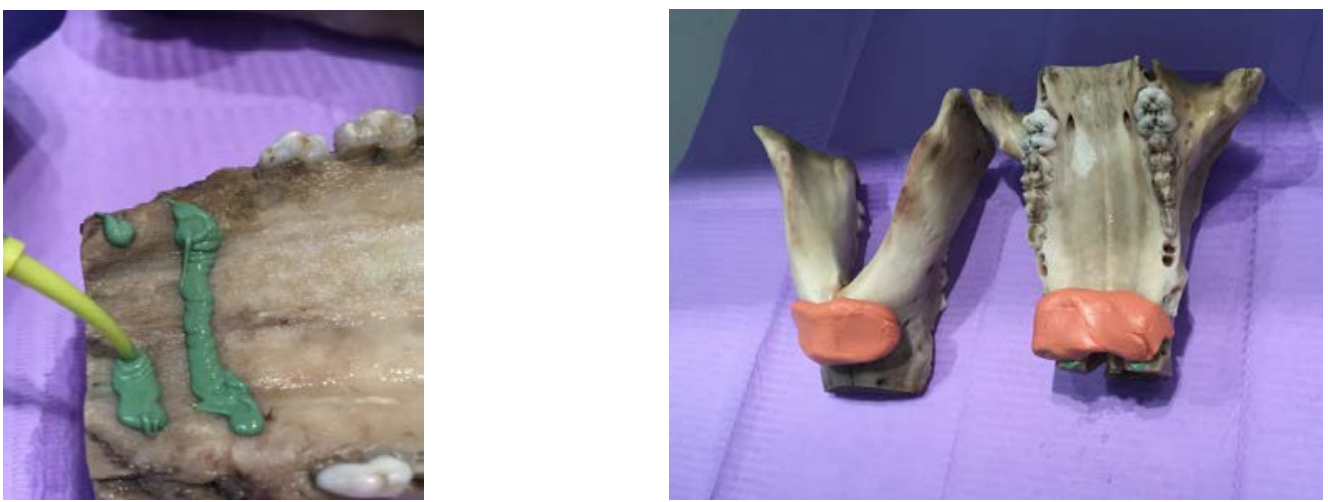

Fig.2 Silicone impressions of the simulated lessions

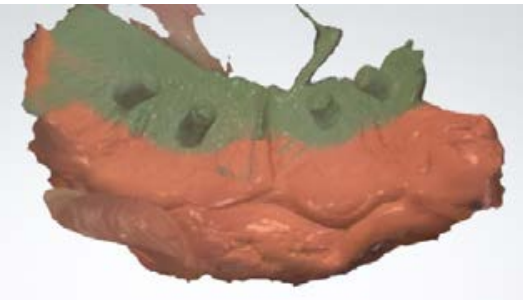

a.

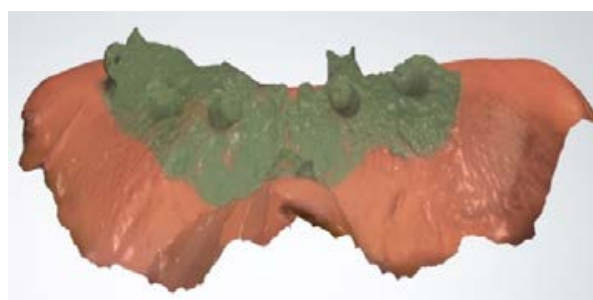

b.

Fig.3 Scanned with IOS silicone impressions of mandibular (a) and maxillary simulated lesions (b)
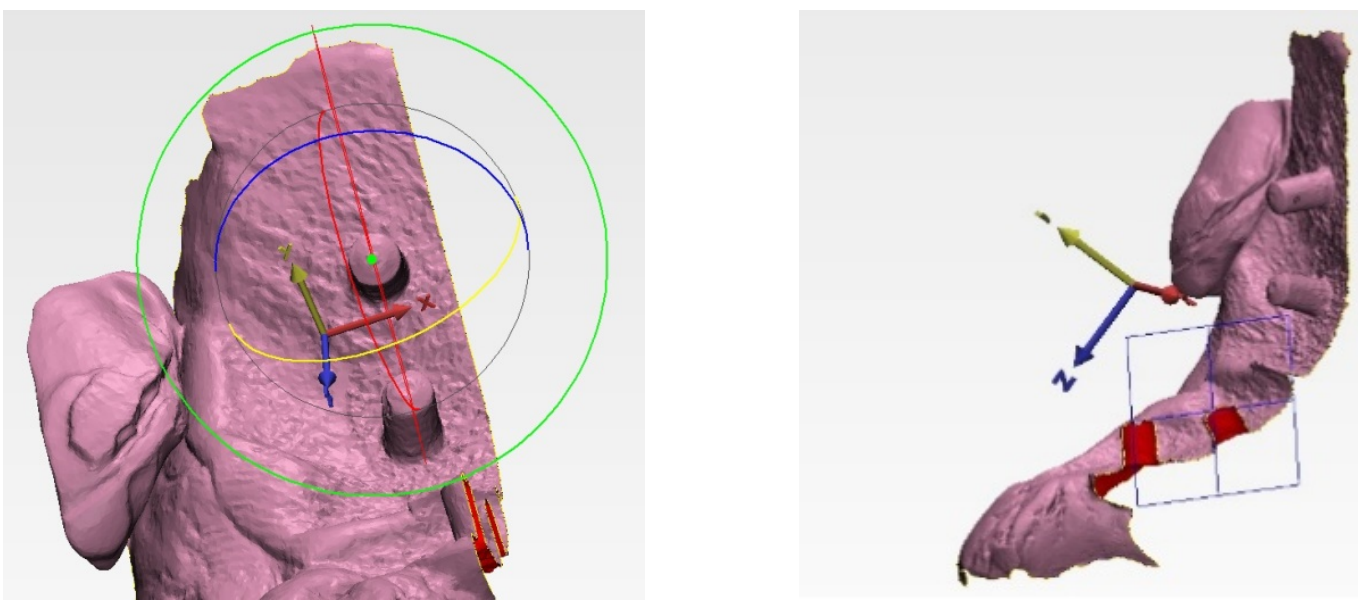

Fig.4 Linear measurements of diameter and depth on the scanned with IOS silicone impressions 

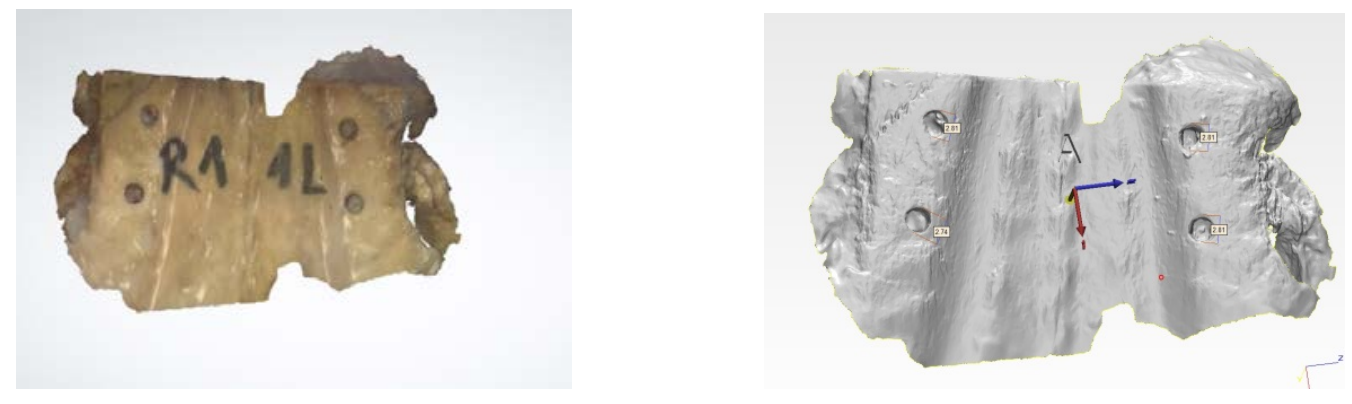

Fig. 5 Digital impression from maxillary simulated lesions (a) and linear measurements (b)

\begin{abstract}
Aim
The aim of our study was to evaluate the accuracy of linear mesurements performed on digitalized and silicone impressions with IOS with the results obtained from direct manual measurements with digital caliper.
\end{abstract}

\title{
Results
}

One way ANOVA and multiple pair comparisons using the Holm-Sidak method showed that there were no significant differences between the diameter of both mandibular and maxillary lesions, measured with IOS (F3sh) and those with the digital caliper at level of significance $\alpha=0.05$. For depth, we found a difference only for maxillary lesions.

The correlation in the diameter values measured with IOS and with a digital caliper was very weak for maxillary and absent for mandibular lesions. For depth, a strong positive correlation was found for mandibular lesions and a weak correlation for maxillary ones. Bland-Altman analysis showed that the lowest mean difference was between the diameter in $M x(0.02 \mathrm{~mm})$, followed by that of diameter $\mathrm{Md}$ $(0.04 \mathrm{~mm})$. There were statistically significant differences in depth Md and Mx (Fig.7). The width of the $95 \%$ confidence interval in the comparison of the feature "diameter" measured using the two methods (F3Sh and $\mathrm{Fi}$ ) is approximately twice as narrow as the respective interval for the "depth" feature, which makes its measurement more accurate than that of depth. For diameter Md (Fig.6) and depth Mx, we established a trend when the feature had low values measured with digital caliper, the F3sh value was higher than $\mathrm{Fi}$, and vice versa for high values of the feature. 


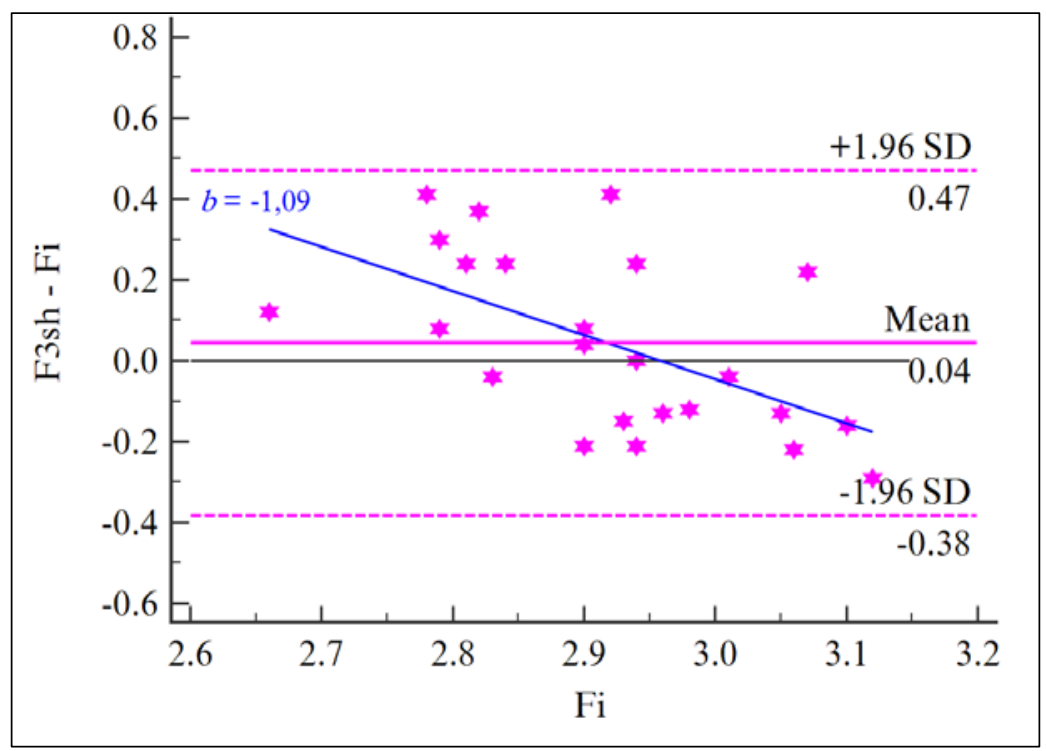

Fig. 6 Bland-Altman plot - difference against the direct measurement (Fi) of the diameter measurement of mandibular lesions on digital impression with IOS (F3sh) and direct measurement with caliper (Fi)

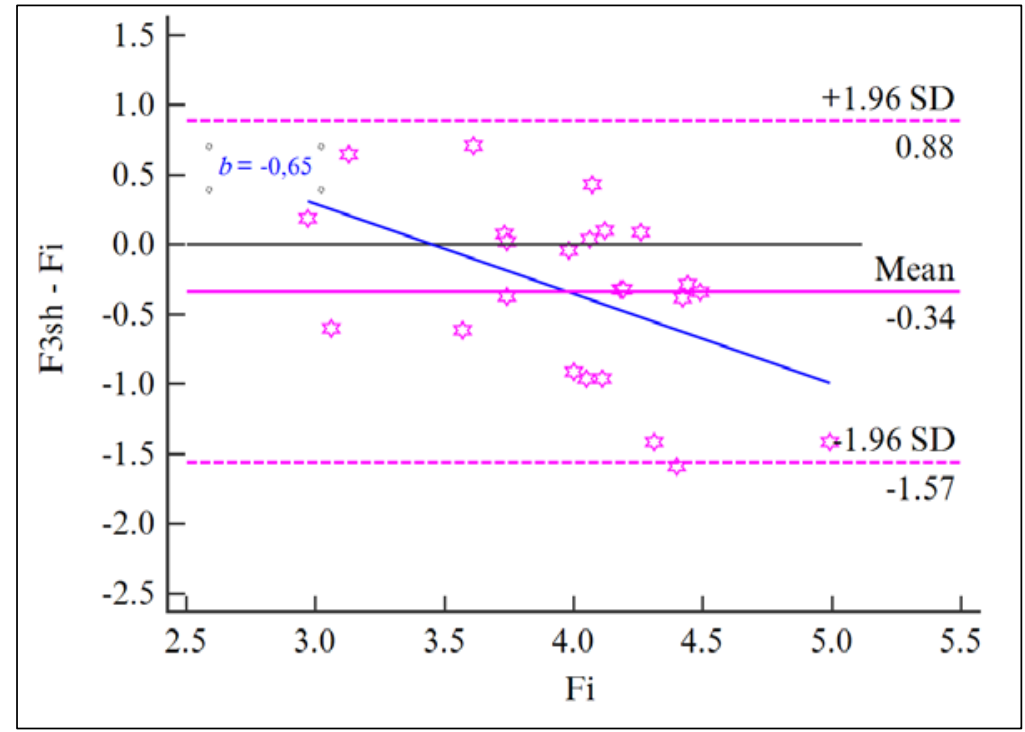

Fig.7 Bland-Altman plot - difference against the direct measurement (Fi) of the depth of maxillary simulated lesions on digital impression with IOS (F3sh) and direct measurement with caliper (Fi) 
An analysis was carried out of the frequency of deviations (in numbers and \%) of diameters measured with F3sh compared to $\mathrm{Fi}$, of $0.5 \mathrm{~mm}$ intervals (Fig. 40), and of absolute deviations with clinical significance criterion with threshold $0.5 \mathrm{~mm}$ and $1 \mathrm{~mm}$.

One way ANOVA and Holm-Sidak multiple pair comparisons showed significant differences between the diameter of mandibular lesions measured on silicone impressions (Fsi) and those obtained with a digital caliper $(F i)$, at level of significance $\alpha=0.05$. The Friedman test did not show differences along the mean values of the diameter of maxillary lesions measured on scanned silicone impressions compared to that with the digital caliper (Fi). For depth, we found a difference between the lesions in both jaws.

The correlation in the variation of diameter values using the two methods was weak for both jaws, and for depth, the correlation coefficient was close to zero. Bland-Altman showed that the lowest mean difference was in diameter $M \times(0.06 \mathrm{~mm})$. The width of the $95 \%$ confidence interval in the comparison of the diameter values measured using the two methods is almost twice as narrow as that for depth.

An analysis of the frequency of deviations (in numbers and in \%) was carried out for diameters measured with Fsi compared to $\mathrm{Fi}$, of $0.05 \mathrm{~mm}$ intervals, as well as of absolute deviations at clinical significance criterion with threshold $0.5 \mathrm{~mm}$ and $1 \mathrm{~mm}$.

\section{Discussion}

The descriptive statistics showed that the mean difference was $0.02 \mathrm{~mm}$ and $0.04 \mathrm{~mm}$ for the mandibular $(\mathrm{Md})$ and maxillary $(\mathrm{Mx})$ lesions, respectively. Mack et al. (1) report a mean difference of -0.17 and -0.13 for the two IOS they used. In our study, in addition to this difference being with a positive sign, i.e. the values measured with 3Shape were systematically higher than those measured with digital caliper $\mathrm{Fi}, \mathrm{a}$ significantly lower value of the difference is observed. For lesion depth, our results are closer to those of Mack - the mean difference was $-0.20 \mathrm{~mm}$ for the lower jaw and $-0.34 \mathrm{~mm}$ for the upper, or, in other words, the values measured with IOS are systematically lower than those from the manual measurement with caliper accepted as the gold standard. Stevens et al. (2) report that the mandibular arch in the anterior section measured with caliper on plaster models was $0.40 \mathrm{~mm}$ longer than that of the digital model, which did not apply to the overall measurement of the same arch from molar to molar. Mullen et al. (3) found significant differences in the measurements of the dental arch's length measured in plaster models and digital models, of up to $1.5 \mathrm{~mm}$ (4). This is in agreement with our finding that, as the value of a feature in the Bland-Altman analysis increases, the difference between the studied features also increases. The authors found statistically significant differences between the length of the mandibular arch measured in digital and in plaster models (Fig.6).

We found a strong correlation for depth of maxillary lesions $(\mathrm{Mx})$ measured with IOS and with caliper. The Bland Altman analysis for comparison of measurements (F3Sh and Fi) for each feature, separately for the maxilla and the mandible, showed a trend so that when a feature has low values (for example, depthFi), the F3Sh measurement is bigger than that with a digital caliper $\mathrm{Fi}$, whereas for higher values of the feature, the F3sh is smaller than those from direct measurement Fi. Our results contradict those of Mack et al. (1), who did not find a proportional difference for any comparison by pairs of measurements. In order to compare the differences in the different features (diameter, depth, volume), as well as assess the 
reliability of the different physical methods of measuring simulated lesions, we calculated the absolute and the percentage difference between measurements in anatomical samples F3Sh scanned with IOS and those with digital caliper Fi. The analysis showed that the absolute difference for diameter measured with F3Sh is lower in value than that for measurements of depth. The lowest percentage difference for diameter was registered in $\mathrm{Md}-4.6 \%$, and for depth of lesions - in $\mathrm{Mx}-10.55 \%$. This means that the value in F3Sh can vary up to $4.6 \%$ and $10.55 \%$ from the Fi value. In other words, at mean value of diameter Md equal to $2.92 \mathrm{~mm}$ and measured with digital caliper, the values obtained with optical scanner vary from it by $0.29 \mathrm{~mm}$ on average, and such a submillimeter difference demonstrates great similarity between the two measurements. It is much more marked in horizontal measurements than in vertical ones. This discrepancy can be partially explained by the high degree of precision with which the contours of the diameter of a given lesion were defined for the purpose of being measured on the digital model obtained with IOS, whereas for depth, we experienced certain difficulties and do not exclude subjectivity in the marking of the points of measurement. In applying IOS in clinical conditions, our results would be influenced by accompanying factors (presence of saliva, soft tissue). The differences in the light beam received back by the scanner can significantly affect its ability to define the actual depth of a given object $(1,5)$.

The accuracy of IOS has been studied by a number of researchers $(1,6,7,8,9,10,11,12,13)$, but we have not found studies of the accuracy of silicone impressions used as a physical method to replace direct osteometry. Silicone impressions of simulated lesions or empty alveoli have been used as the gold standard by few researchers $(14,15)$.

Our study aimed to examine the accuracy and reliability of silicone impressions as a physical measurement method in the analysis of the accuracy of linear and volumetric CBCT measurements. When silicone was used for positive impressions of jaw lesions (or dental alveoli), the volume was calculated following the principle of calculating the displaced volume of water in a graduated glass vessel or the mathematical formula for calculating the volume of a geometric shape, such as a sphere (16). We scanned the silicone impressions with IOS and carried out our measurements on the obtained threedimensional images. The methodology of creating simulated lesions in the jaws used in our study provided full symmetry of the lesion walls, absence of deviation in the values of depth measured medially and distally in the lesion and sharp borders. In contrast to other researchers who have used silicone for manual measurements, we did not simply compare the results from the measurements using this method with the values obtained from measurements on the radiographic images from CBCT, but we also compared the accuracy of the measurements on the scanned silicone with those from direct measurement with digital caliper. With view of analyzing the clinically significant accuracy of the measurements, we calculated the percentage distribution of differences between the values of the features "diameter" and "depth" obtained from the scanned silicone impressions (Fsi) and with digital caliper (Fi). Using a threshold of $0.5 \mathrm{~mm}$ to determine several degrees of "clinically significant accuracy", $62 \%$ of the measurements of diameter of $\mathrm{Md}$ lesions with Fsi were from 0 to $0.5 \mathrm{~mm}$ lower than the diameter measured in direct osteometry with digital caliper $\mathrm{Fi}$ (i.e., from 0 to $-0.05 \mathrm{~mm}$ ), whereas for the same feature in $\mathrm{Mx}$, this percent was $45 \%$. Conversely, in the range from 0 to $0.5 \mathrm{~mm}$, the percentages were $29.2 \%$ for Md and $50 \%$ for $\mathrm{Mx}$, or these are the cases where Fsi obtained a higher value than Fi. The comparison of the diameters of mandibular simulated lesions showed systematically lower values from Fsi than from $\mathrm{Fi}$ (Mean $-0.13 \mathrm{~mm}$ ), whereas for Mx diameter, they were systematically higher even though the Mean value was very close to $0(0.06 \mathrm{~mm})$. For the depth of the lesions, in addition to systematically lower values for both jaws (Mean/Md=-0.35, Mean/Mx=-0.30), a distribution of the frequency of deviation of Fsi from $\mathrm{Fi}$ is 
observed in all degrees of "clinical inaccuracy". This makes the measurement of the lesion diameter on scanned silicone impression more accurate than the measurement of their depth. We found the same in the comparison of the accuracy of diameter and depth measurements when IOS is used for scanning the anatomical samples.

\section{Conclusion}

Scanning anatomical samples with IOS is a reliable method for objectivizing the anatomical truth in studies of the accuracy of CBCT measurements.

Silicone impressions of simulated defects in jaws used for studying the accuracy of CBCT measurements are not a recommended method for objectivizing the anatomical truth.

\section{References}

1. Mack S, Bonilla T., English J., cozad B., Akyalcin S., Accuracy of 3-dimensional curvilinear measurements on digital models with intraoral scanners, Am.Journal of Orthodontics and Dentofacial Orthopedics, volume 152, Issue 3, p.42-425

2. Stevens DR, Flores-Mir C, Nebbe B, Raboud DW, Heo G, Major PW. Validity, reliability, and reproducibility of plaster vs digital study models: comparison of peer assessment rating and Bolton analysis and their constituent measurements. Am J Orthod Dentofacial Orthop 2006;129:794-803.

3. Mullen SR, Martin CA, Ngan P, Gladwin M. Accuracy of space analysis with eModels and plaster models. Am J Orthod Dentofacial Orthop 2007;132:346-52.

4. Redlich M, Weinstock T, Abed Y, Schneor R, Holdstein Y, Fischer A. A new system for scanning, measuring and analyzing dental casts based on a 3D holographic sensor. Orthod Craniofac Res. 2008; 11:90-5.

5. Akyalcin S, Cozad BE, English JD, Colville CD, Laman S. Diagnostic accuracy of impression-free digital models. Am J Orthod Dentofa- cial Orthop 2013;144:916-22.

6. Sousa MV, Vasconcelos EC, Janson G, Garib D, Pinzan A. Accuracy and reproducibility of 3dimensional digital model measurements. Am J Orthod Dentofacial Orthop 2012;142:269-73.

7. Wiranto MG, Engelbrecht WP, Tutein Nolthenius HE, van der Meer WJ, Ren Y. Validity, reliability, and reproducibility of linear measurements on digital models obtained from intraoral and conebeam computed tomography scans of alginate impressions. Am J Orthod Dentofacial Orthop 2013;143:140-7.

8. Grunheid T, McCarthy SD, Larson BE. Clinical use of a direct chairside oral scanner: an assessment of accuracy, time, and patient acceptance. Am J Orthod Dentofacial Orthop 2014; 146:673-82.

9. Aragon ML, Pontes LF, Bichara LM, Flores-Mir C, Normando D. Validity and reliability of intraoral scanners compared to conven- tional gypsum models measurements: a systematic review. Eur J Orthod 2016;38:429-34.

10. Naidu D, Freer TJ. Validity, reliability, and reproducibility of the iOC intraoral scanner: a comparison of tooth widths and Bolton ra- tios. Am J Orthod Dentofacial Orthop 2013;144:304-10.

11. Flugge TV, Schlager S, Nelson K, Nahles S, Metzger MC. Precision of intraoral digital dental impressions with iTero and extraoral digitization with the iTero and a model scanner. Am J Orthod Den- tofacial Orthop 2013;144:471-8.

12. Seelbach P, Brueckel C, Wo€stmann B. Accuracy of digital and con- ventional impression techniques and workflow. Clin Oral Investig 2013;17:1759-64. 
13. Nedelcu RG, Persson AS. Scanning accuracy and precision in 4 in- traoral scanners: an in vitro comparison based on 3-dimensional analysis. J Prosthet Dent 2014;112:1461-71.

14. Ahlowalia M. S., S. Patel, H. M. S. Anwar, G. Cama, R. S. Austin, R. Wilson \& F. Mannocci Accuracy of CBCT for volumetric measurement of simulated periapical lesions Oral Surgery, Oral Medicine, Oral Pathology, Oral Radiology \& Endodontology. Jan2008, Vol. 105 Issue 1, p91-98. $8 p$.

15. Liang Y.-H., Jiang L., Gao X.-J., Shemesh H., Wesselnik P.R., Wu M.-K., Detection and measurement of artificial periapical lesions by cone-beam computed tomography, International Endodontic Journal, 47, 332-338, 2014

16. Patel A., Tee B., Fields H., Jones E., Jahanzeb Chaudhry J., Sun Z., Evaluation of cone-beam computed tomography in the diagnosis of simulated small osseous defects in the mandibular condyle, February 2014, Vol 145, Issue 2 American Journal of Orthodontics and Dentofacial Orthopedics, p.143-56

\section{Corresponding author:}

Dora Kishkilova,

Department of Diagnostic Imaging

and Oral Diagnostic,

Faculty of Dentistry,

Medical University - Sofia

1.Georgi Sofijski Blvd,

Sofia, Bulgaria

Tel.: +359887 777807 